\title{
Nanoblocks embedded in L-shaped nanocavity of a plasmonic sensor for best sensor performance
}

\author{
M.A. ButT ${ }^{1,2 *}$, N.L. KAZANSKIY ${ }^{1,3}$ \\ ${ }^{1}$ Samara National Research University, 443086 Samara, Russia \\ ${ }^{2}$ Warsaw University of Technology, Institute of Microelectronics and Optoelectronics, \\ Koszykowa 75, 00-662 Warszawa, Poland \\ ${ }^{3}$ Institute of RAS - Branch of the FSRC “Crystallography and Photonics” RAS, Samara, Russia \\ *Corresponding author: butt.m@ssau.ru
}

\begin{abstract}
In this work, we proposed a highly sensitive design of a plasmonic sensor which is formed by embedding a periodic array of nanoblocks in L-shaped cavity formed by the metal-insulator-metal waveguide. The nanoblocks are placed in the strong electric field confinement region to further enhance its strength by confining it to a small area. To validate the study, the spectral characteristics of the proposed sensor design is compared to the spectral characteristics of a standard design having the same geometric parameters excluding nanoblocks in the cavity. The study shows that the incorporation of 5 nanoblocks of length $25 \mathrm{~nm}$ in the cavity can provide best performance indicators in the form of sensitivity, figure of merit and $Q$-factor. The sensitivity, figure of merit and $Q$-factor of the proposed sensor design is $1065 \mathrm{~nm} / \mathrm{RIU}, 251.17$ and 343.4 which is significantly higher than the standard L-shape resonator design. The sensor design can be developed with a single fabrication step. Due to the ease of fabrication and the highly responsive nature of the design, it can be used in biomedical applications.
\end{abstract}

Keywords: plasmonic sensor, refractive index sensor, metal-insulator-metal waveguide, L-shaped cavity, finite element method.

\section{Introduction}

Surface plasmon polaritons (SPPs) are a kind of a charge density waves that propagate along with the metal-insulator interface and have been extensively researched due to their ability to confine the light in the subwavelength region in addition to overcoming the diffraction limit $[\underline{1}, \underline{2}]$. Several plasmonic devices have been proposed in recent

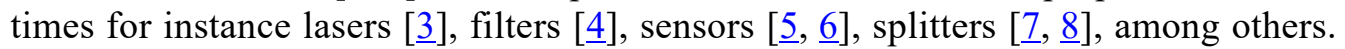
Due to extraordinary spectral characteristics and miniaturized footprint, plasmonic sensors have gathered extra consideration compared to traditional optical sensors based on silicon platforms or optical fibre sensors [9]. The sensitivity offered by a plasmonic sensors is several orders of magnitude higher than conventional sensors and their small size allows an easy integration of devices with the bulk. 
Metal-insulator-metal (MIM) waveguide scheme is one of the most widely used plasmonic-based nanostructures for the comprehension of integrated optical circuits. The waveguide structure is formed by enclosing an insulator (can be air, $n=1.0$ ) between two metal claddings [10]. The straightforward design and proficiency of these waveguides to confine light at the subwavelength level are the key attributes of this waveguide system. In the last decade, several plasmonic sensors such as gas sensor [11], temperature sensor [12], refractive index sensor [13] have been proposed based on this waveguide scheme which exhibits exceptional performance.

High sensitivity $(S)$ and figure of merit (FOM) are required to guarantee the outstanding performance of the sensor with high optical resolution. There has been a great deal of effort to improve $S$ on MIM plasmonic sensors, but longer wavelength always possesses a broader full width at half maximum (FWHM), which results in lower FOM. Fano resonance can be one of the possible solutions which arise due to the interference between a discrete quantum state and continuum band of states resulting in an asymmetric spectral line shape with narrower FWHM. This significantly improves the FOM. However, such resonance is unstable and can be simply broken due to a mismatch in phase or mode that could be caused by localized structural degradation or alteration in a localized refractive index.

Several novel MIM plasmonic sensor designs have been recently proposed for refractive index sensing applications, for instance: a double-square cavity refractive index sensor has been suggested for the simultaneous detection of human blood group offering the sensitivity and FOM of $1380 \mathrm{~nm} / \mathrm{RIU}$ and 104, respectively [14]. LI et al . proposed a tunable plasmonically induced reflection in HRR-coupled MIM waveguide structure which offers the sensitivity of $753 \mathrm{~nm} / \mathrm{RIU}$ [15]. A notched ring resonator is designed by YAN et al. which provides the sensitivity and FOM of $1071.4 \mathrm{~nm} / \mathrm{RIU}$ and 14.29 , respectively [16].

Nevertheless, in our previous works [17-21], we presented several novel plasmonic sensor designs based on MIM waveguide. Those designs are highly sensitive but the FOM and $Q$-factor were quite moderate. In this work, we proposed a highly sensitive refractive index sensor design with a high FOM and $Q$-factor that is easy to manufacture in a few single fabrication steps and can be utilized in biochemical applications. The study is carried out via a commercial finite element (FEM) simulation tool (COMSOL Multiphysics 5.1). In COMSOL simulations, the entire sensor design is divided into triangular mesh element with $\lambda / 10$ grid size, which provides the precise simulation results within the available computational resources. A computational domain with open boundaries is modelled that allows the electromagnetic wave to pass without reflection. To approximate an open geometry, scattering boundary conditions (SBC) are used at the outer edges of the FEM simulation window.

\section{Standard L-shaped cavity sensor design}

In this section, we proposed a plasmonic sensor design based on MIM waveguide. The design configuration is composed of an L-shaped cavity side coupled to a MIM bus 


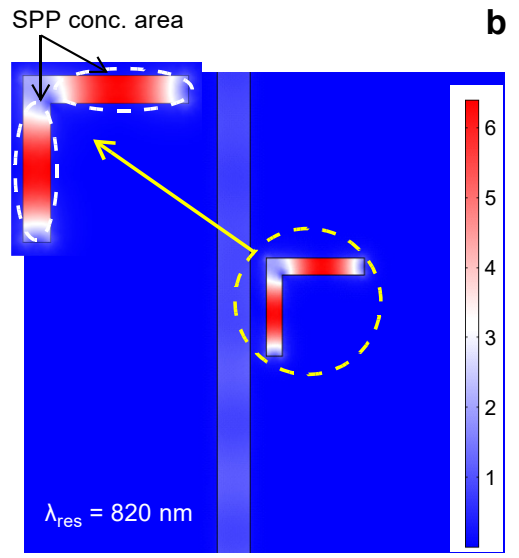

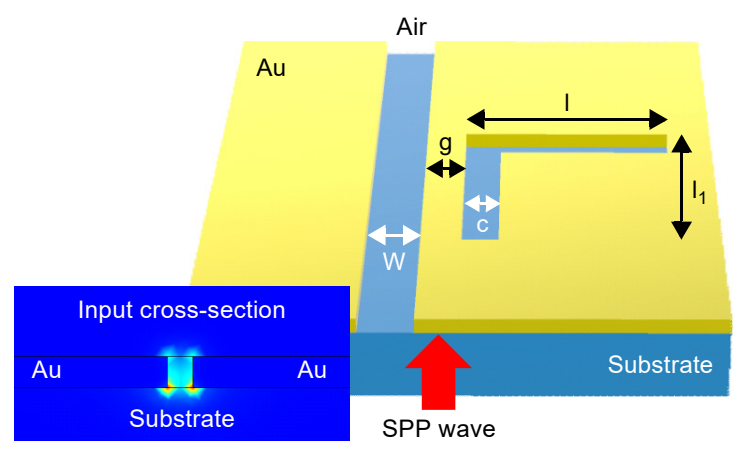

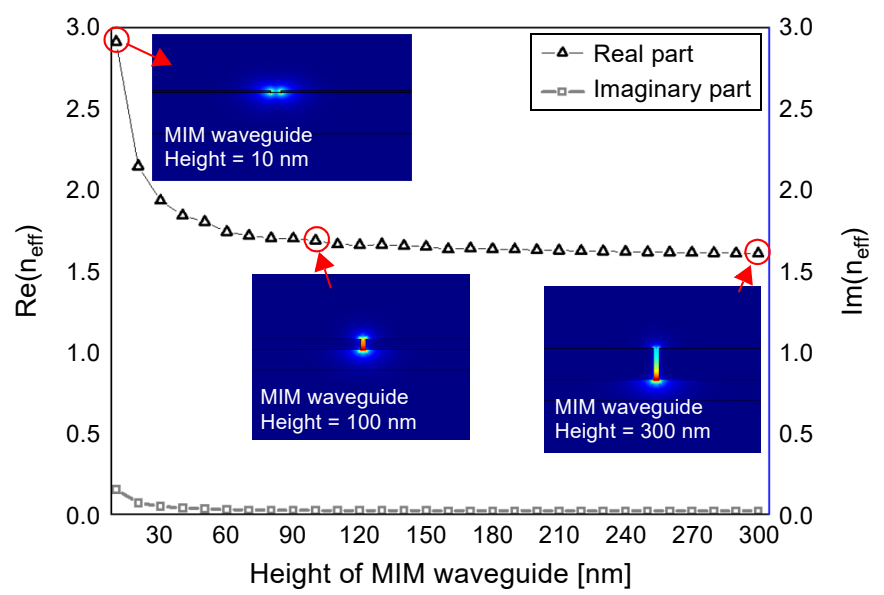

C

Fig. 1. Schematic of a standard MIM resonator design. Inset shows the $E$-field distribution at the input of the MIM waveguide (a). E-field distribution in the cavity at resonance wavelength (b). The relationship between the height of the MIM waveguide and effective refractive index of the SPPs (c).

waveguide. The width of the bus waveguide is denoted as $W$. The side lengths of the cavity are represented as $l$ and $l_{1}$ which are equal in both vertical and horizontal direction. The width of the cavity and the gap between the bus waveguide and cavity is labelled as $c$ and $g$, respectively. The $W$ and $c$ are fixed at 100 and $50 \mathrm{~nm}$ throughout the paper. The schematic of the sensor design is shown in Fig. 1a.

The metal and dielectric material are selected as gold ( $\mathrm{Au}$ ) and air, respectively. $\mathrm{Au}$ is preferred as the metal layer due to its biocompatibility and resistance to oxidation compared to silver. The refractive index of $\mathrm{Au}$ is calculated from the Drude-Lorentz model [22]:

$$
\varepsilon=\varepsilon_{\infty}-\frac{\omega_{\mathrm{p}}^{2}}{\omega^{2}+j \omega \gamma}
$$


where $\varepsilon_{\infty}=9.0685, \omega_{\mathrm{p}}=135.44 \times 10^{14} \mathrm{rad} / \mathrm{s}$ and $\gamma=1.15 \times 10^{14} \mathrm{rad} / \mathrm{s}$. The MIM bus waveguide is excited by a TM polarized plane wave. As $W \ll \lambda_{\text {incident }}$, therefore, merely TM fundamental mode can exist. The dispersion relation of the fundamental mode can be written as:

$$
\begin{aligned}
& \frac{\varepsilon_{\mathrm{d}} p}{\varepsilon_{\mathrm{m}} k}=\frac{1-\exp (k W)}{1+\exp (k W)} \\
& k=k_{\mathrm{o}} \sqrt{\left(\frac{\beta_{\mathrm{SPP}}}{k_{\mathrm{o}}}\right)^{2}-\varepsilon_{\mathrm{d}}} \\
& p=\sqrt[k]{\left(\frac{\beta_{\mathrm{SPP}}}{k_{\mathrm{o}}}\right)^{2}-\varepsilon_{\mathrm{m}}} \\
& \beta_{\mathrm{SPP}}=n_{\mathrm{eff}} k_{\mathrm{o}}=n_{\mathrm{eff}} \frac{2 \pi}{\lambda}
\end{aligned}
$$

Here $W$ is the width of the bus waveguide and $\lambda$ is the wavelength of the incident light in vacuum, $\varepsilon_{\mathrm{m}}$ and $\varepsilon_{\mathrm{d}}$ are the relative permittivity of metal and dielectric, $\beta_{\mathrm{SPP}}$ and $n_{\text {eff }}$ are the propagation constant and effective index of SPPs, and $k_{\mathrm{o}}=2 \pi / \lambda$ is the wave number. A polychromatic light source, a halogen lamp or a superluminescent diode (SLED) can be used covering the entire spectrum in which $\lambda_{\text {res }}$ is supposed to occur. Halogen lamp technology is an ideal candidate due to its broad light spectrum. The $E$-field distribution in the L-shaped cavity at $\lambda_{\text {res }}$ is shown in Fig. $1 \mathbf{b}$. The inset of the figure shows the areas in the cavity where the $E$-field is highly concentrated.

The majority of the previous studies conducted on plasmonic sensors have utilized 2D numerical simulations, which treat one dimension as infinity. This facilitates the analysis of the sensor performance with much shorter computational time and lower loss [23] , and this is done in this work. However, the height of the MIM waveguide has an important effect on the loss of the system, which has to be taken into consideration for practical processing. Figure 1c shows the relationship between the $n_{\text {eff }}$ and the height of the MIM waveguide which is simulated for $850 \mathrm{~nm}$ wavelength. The gap between the metal rails is $50 \mathrm{~nm}$ which is just air. As the height of the waveguide increases, the modal power at the metal interface decreases which results in the gradual decay of the real and imaginary part of the $n_{\text {eff }}$. The reduction in $\operatorname{Im}\left(n_{\text {eff }}\right)$ signifies that the system has low loss which can result in the realization of the waveguides with longer propagation length. The $n_{\text {eff }}$ stabilizes when the height of waveguide is greater than $\sim 50 \mathrm{~nm}$ which shows that the height of the waveguide does not affect the $n_{\text {eff }}$ after a certain value. The $n_{\text {eff }}$ obtained in 3D simulation agrees with the solution obtained via $2 \mathrm{D}$ simulation. Even if the height of the waveguide approaches infinity, the $n_{\text {eff }}$ stays the same which is the case of a $2 \mathrm{D}$ simulation. 

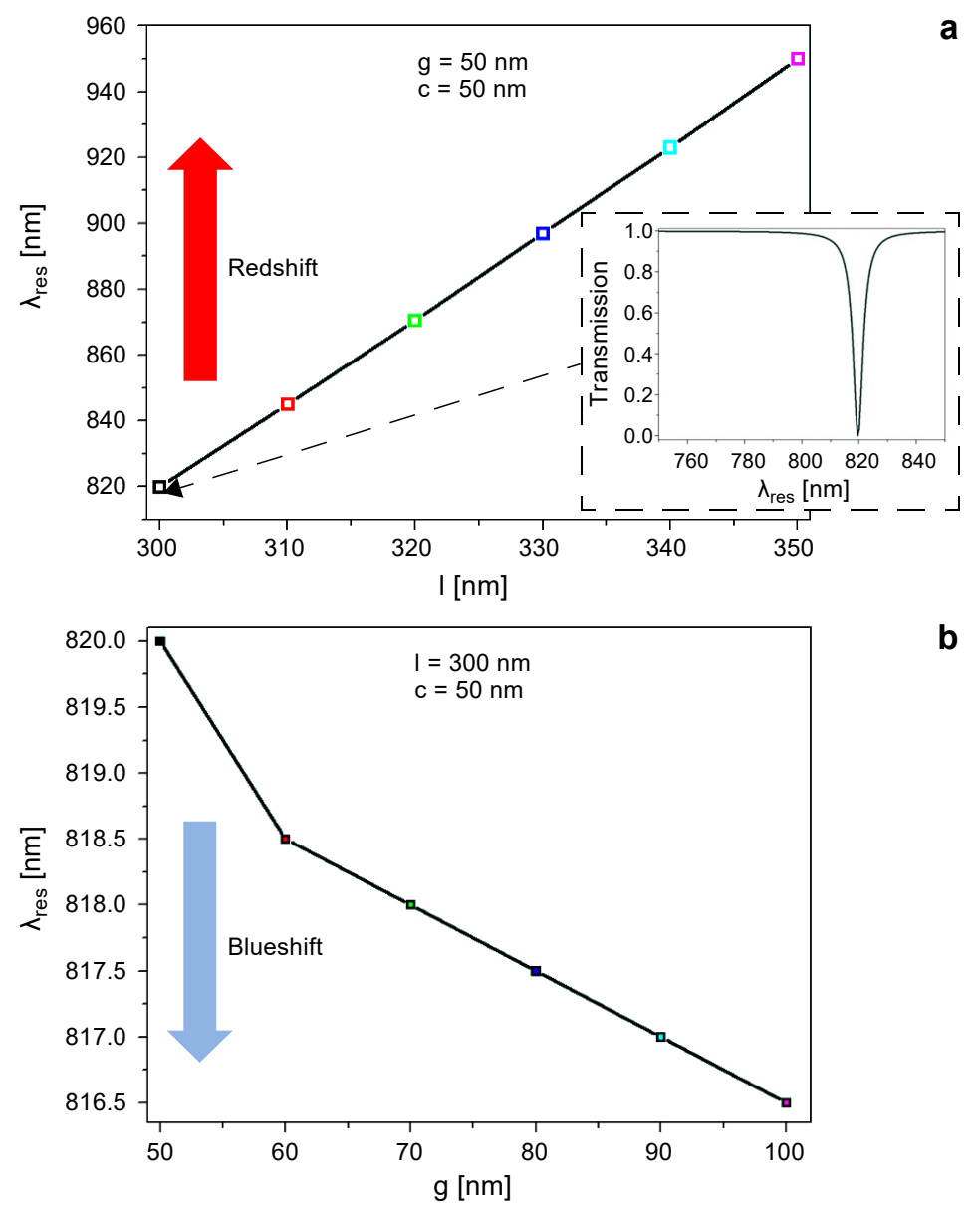

b

Fig. 2. Resonance wavelength dependence on the geometric parameters of the sensor design. (a) Side length $l$, (b) gap $g$ between the bus waveguide and the sensor cavity.

Here, the effect of sidelength $\left(l\right.$ and $\left.l_{1}\right)$ on the resonance wavelength $\left(\lambda_{\text {res }}\right)$ is studied by maintaining the $g$ and $c$ at $50 \mathrm{~nm}$. Three different combinations of $l$ and $l_{1}$ are studied: $l_{1}>l, l_{1}<l$ and $l=l_{1}$. In all three combinations, the $\lambda_{\text {res }}$ performs a redshift with either an increase in $l$ or $l_{1}$. Here $l=l_{1}$ case is discussed where $l$ is varied between 300 and $350 \mathrm{~nm}$. With an increase in $l$, the $\lambda_{\text {res }}$ shows a large redshift. After the fabrication process, the sensor should be carefully calibrated to trace the $\lambda_{\text {res }}$ because $\pm 50 \mathrm{~nm}$ of fabrication inaccuracy can bring a $\lambda_{\text {res }}$ shift of $\sim 120 \mathrm{~nm}$ as can be seen in Fig. 2a. Inset of the figure shows a sharp Lorentzian dip at $\lambda_{\text {res }}=820 \mathrm{~nm}$ when $l=300 \mathrm{~nm}$. The full width at half maximum (FWHM) of the dip is $\sim 4.29 \mathrm{~nm}$. In Fig. $2 \mathbf{b}$, the relationship between $\lambda_{\text {res }}$ and $g$ is presented, which shows the weak control of $g$ on $\lambda_{\text {res }}$. The calculation shows the flexibility of selecting the distance between the cavity and the bus 
waveguide because $\Delta g=50 \mathrm{~nm}$ can only bring a $\Delta \lambda_{\text {res }}=3.5 \mathrm{~nm}$. Therefore, the parameter $g$ has a high fabrication inaccuracy tolerance.

The fabrication of the proposed sensor design is straightforward and can be achieved in a few simple steps. First of all, a thin layer of gold is deposited on a silica substrate followed by $E$-beam lithography patterning of the design. The main advantage of $E$-beam lithography is due to its ability to engrave custom patterns with sub-10 nm resolution which makes it possible to embed nanoblocks (NBs) of $10-25 \mathrm{~nm}$ in the cavity. MIM waveguides are obtained by removing the unwanted parts of the metal layer from the design with the help of chemical etching. One possible method is the use of diluted nitric acid and water which offers high etch selectivity.

\section{Nanoblocks embedded in L-shaped cavity sensor design}

In this section, we improved the sensing performance of the device by the amplification of SPPs in the resonator cavity. This modification is carried out by placing an array of
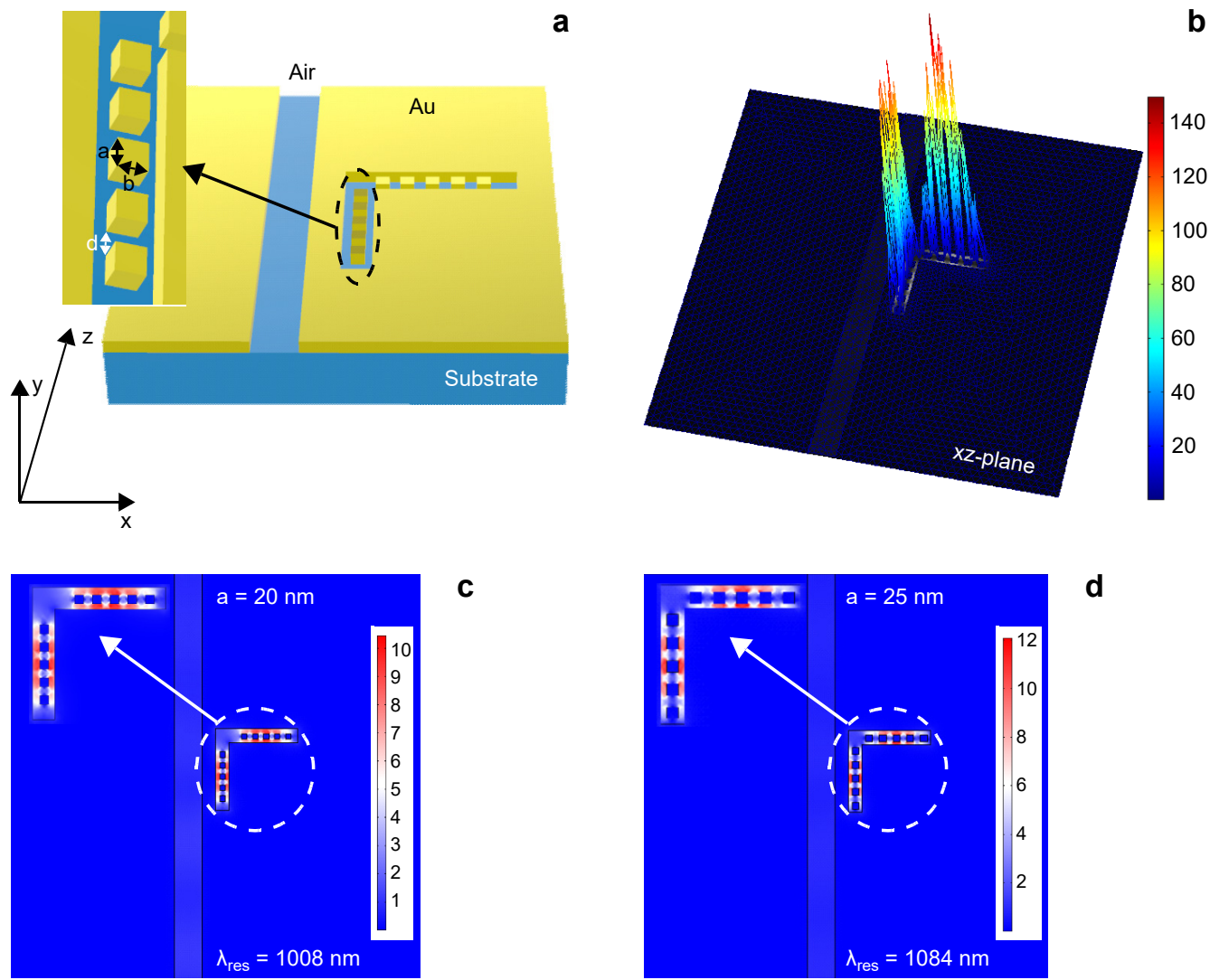

Fig. 3. Schematic of NBs embedded MIM resonator design. Inset shows the array of NBs separated by a distance $d$ (a). 3D profile of SPP confined in the cavity at $\lambda_{\text {res }}=1008 \mathrm{~nm}(\mathbf{b}) . E$-field distribution in the cavity embedded with NBs at resonance wavelength length $a=20 \mathrm{~nm}(\mathbf{c})$, and $a=25 \mathrm{~nm}(\mathbf{d})$. 
nanoblocks (NBs) in the cavity where a high concentration of electric field is present as labelled in the inset of Fig. $1 \mathbf{b}$. The NBs are separated by a distance $d$ which is equal to the side length of the NB. The side length of NB is denoted by $a$ and $b$ and NB is in the form of a square. Therefore, we are considering $a=b$. The schematic of the proposed sensor design is shown in Fig. 3a. We selected 5 NBs of different sizes $(5-25 \mathrm{~nm})$ arranged on both sides of the L-shaped cavity as illustrated in the inset of Fig. 3a. The electric field distribution is plotted when 5 NBs of $a=20 \mathrm{~nm}$ and $a=25 \mathrm{~nm}$ are positioned on both sides of the cavity as shown in Figs. $3 \mathbf{c}$ and $3 \mathbf{d}$, respectively. The colour bar in the figure shows the strength of the electric field which is about twice the value obtained in standard design (see Fig. 1b).

\section{Sensor performance}

In this section, we have discussed three important factors such as sensitivity $(S)$, the figure of merit (FOM) and quality factor ( $Q$-factor) which is used to estimate the performance of the plasmonic sensor. Prior to this, the $n_{\text {eff }}$ of the standard cavity and NBs loaded cavity is plotted over a wavelength region of 800 to $1100 \mathrm{~nm}$ which is the operational wavelength range of our sensor. The $n_{\text {eff }}$ of the NBs loaded cavity design is more than twice times higher than the standard design which shows the presence of higher modal power in the cavity. This is what we have observed in the colour bar of Fig. 3. When the medium refractive index is changed from 1.0 to 1.3 , a large $\Delta n_{\text {eff }}=0.919$ of NBs loaded cavity takes place compared to standard cavity design which is only 0.5225 as shown in Fig. 4a. This signifies the susceptibility of both the sensor designs.

The ring resonators are receptive to the medium and their $\lambda_{\text {res }}$ shows a redshift in the case of a slight change in the ambient refractive index. The ideal and highly responsive sensors should have a large redshift in $\lambda_{\text {res }}$ to the small change in the refractive index of the medium. The performance of the plasmonic sensor is measured in terms

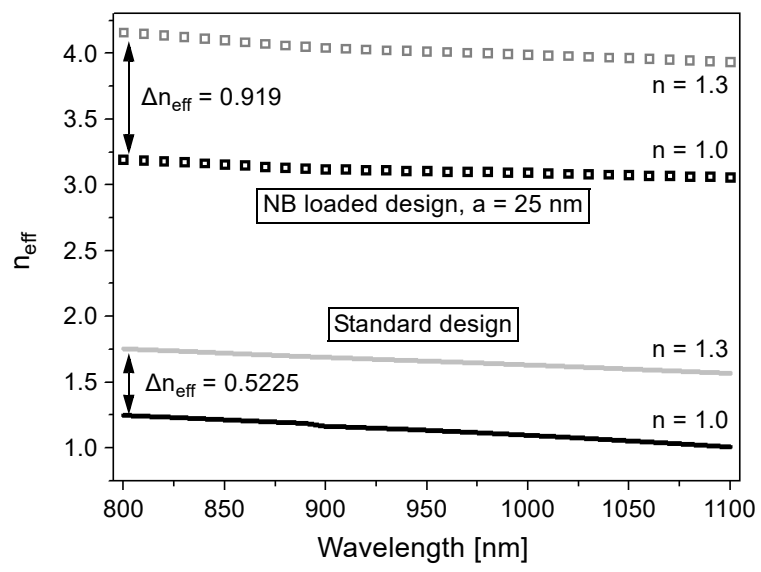

Fig. 4. The $n_{\text {eff }}$ of the sensor designs at ambient refractive index, $n=1.0$ and $n=1.3(\mathbf{a}), S(\mathbf{b})$, and FOM and $Q$-factor (c). 


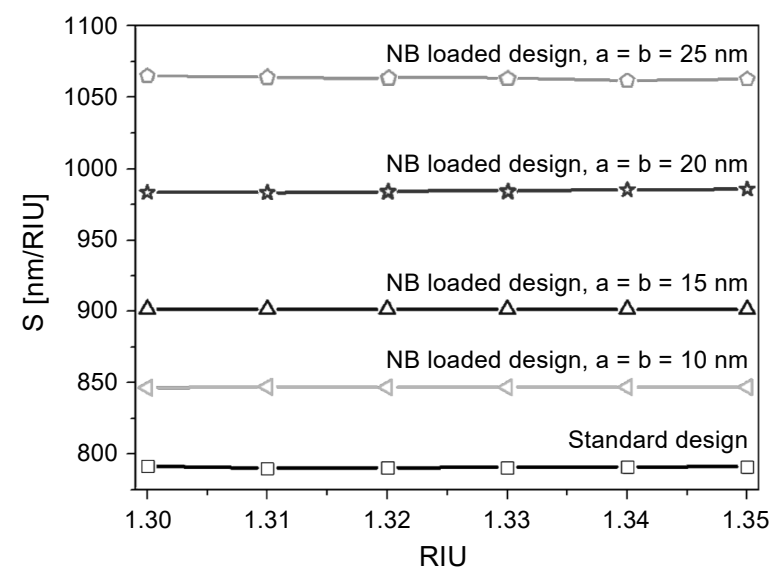

b

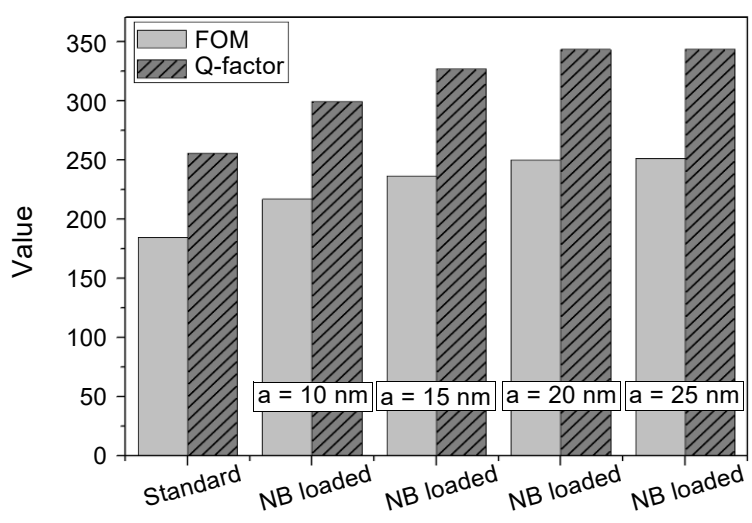

C

Design

Fig. 4. Continued.

of its ability to detect changes in the refractive index [24-26]. This is evaluated as the bulk refractive index sensitivity $S$ and calculated by using the following expression:

$$
S=\Delta \lambda / \Delta n
$$

where $\Delta \lambda$ is the change in the $\lambda_{\text {res }}$ and $\Delta n$ the change in ambient refractive index. The transmission spectrum of the resonator is calculated by filling the medium with refractive index of $1.3,1.31,1.32,1.33,1.34$, and 1.35 which is equivalent to the refractive index of several intra- and extracellular fluids. The spectral characteristics are studied for the sensor design with geometric parameters such as the side length $l$, gap $g$, cavity width $c$ and width of bus waveguide $W$ fixed at 300, 50, 50 and $100 \mathrm{~nm}$, respectively. The size $(a=b)$ of NBs is varied from 10 to $25 \mathrm{~nm}$ in NBs loaded cavity design. The $S$ of the standard and NBs loaded design is shown in Fig. 4b. The standard L-shaped cavity design exhibits an $S$ of $791.67 \mathrm{~nm} / \mathrm{RIU}$ which linearly enhances as NBs are placed in the cavity. The highest $S$ of $1065 \mathrm{~nm} / \mathrm{RIU}$ is obtained when the L-shaped cav- 
T a b 1 e. Sensitivity $S$ and FOM comparison with previously reported values.

\begin{tabular}{lcccl}
\hline References & $S[\mathrm{~nm} / \mathrm{RIU}]$ & FOM or FOM & $Q$-factor & iDL \\
\hline$\underline{21}]$ (our previous work) & 1320 & 16.7 & 18.9 & - \\
{$[\underline{20}]$ (our previous work) } & 1240 & 20 & 20.9 & - \\
{$[\underline{19}]$ (our previous work) } & 1680 & 48 & 48.4 & - \\
{$[\underline{18}]$ (our previous work) } & 793.3 & 82.1 & 52.9 & - \\
{$[\underline{24}]$ (other related work) } & 685 & - & $19-32$ & - \\
{$[\underline{25}]$ (other related work) } & 596 & 7.5 & - & - \\
{$[\underline{26}]$ (other related work) } & 1060 & 176.7 & 145 & - \\
{$[\underline{27}]$ (other related work) } & $416-986$ & $\mathbf{3 . 2} \times \mathbf{1 0}$ & - & - \\
This work - standard design & 791.67 & 184.53 & 255.71 & 0.00405 \\
This work - NBs embedded, $a=10 \mathrm{~nm}$ & 847.41 & 217.28 & 299.61 & 0.00444 \\
This work - NBs embedded, $a=15 \mathrm{~nm}$ & 901.66 & 236.65 & 327.03 & 0.00423 \\
This work - NBs embedded, $a=20 \mathrm{~nm}$ & 985.71 & 250.18 & 343.4 & 0.00401 \\
This work - NBs embedded, $a=25 \mathrm{~nm}$ & 1065 & 251.17 & 343.4 & 0.00398 \\
\hline
\end{tabular}

ity is loaded with $5 \mathrm{NBs}(a=25 \mathrm{~nm})$ on each side. In our design, we have a limitation of incorporating a maximum of $5 \mathrm{NBs}$ due to the small size of the cavity. We believe that the $S$ can be further enhanced by increasing the cavity length, number and size of the NBs.

The optical resolution of the sensor is a vital parameter which should be considered while designing the plasmonic sensors, for that reason a high figure of merit (FOM) is desired. The FWHM of the standard resonator design is $4.29 \mathrm{~nm}$ whereas the FWHM of NBs embedded sensor design is 3.9, 3.81, 3.94 and $4.24 \mathrm{~nm}$ for $a=10,15,20$ and $25 \mathrm{~nm}$, respectively. The narrow FWHM results in a high FOM which is listed in the Table and compared with the previously reported values. We would like to mention that in some works $[27,28]$, the FOM is represented as FOM ${ }^{*}$ which is several times higher in order than the one obtained in this work and is calculated differently using the expression $\Delta R /(R \Delta n)$ at a fixed wavelength, where $\Delta R$ denotes the reflection intensity variation due to the change in the refractive index $\Delta n$ of the surrounding medium and $R$ is the reflection rate in the sensor structure. It can also be calculated by using the expression $\Delta T /(T \Delta n)$, where $T$ denotes the transmittance in the proposed structures and $\Delta T / \Delta n$ is the transmission change at a fixed wavelength induced by a refractive index change. There are several applications related to optical resonators where a high $Q$-factor is desired. These applications include high-performance lasers [29], narrow-bandwidth filters $[\underline{30}, \underline{31}]$ and high-efficiency nonlinear optic devices [32]. The $Q$-factor is defined as $\lambda_{\text {res }} / F W H M$, where $\lambda_{\text {res }}$ is the resonance wavelength and FWHM is the full width at half maximum of the resonance peak. The intrinsic detection limit (iDL) of the proposed sensor designs is also calculated by utilizing the expression [33]: $\mathrm{iDL}=\lambda_{\text {res }} / S Q$ which defines the detection capabilities of $\Delta n$ in the medium. The highest $Q$-factor is obtained for the L-shaped cavity design loaded with $5 \mathrm{NBs}$ of $a=25 \mathrm{~nm}$. The spectral characteristics of the sensor designs discussed in this paper are listed in the Table. 


\section{Conclusion}

We proposed a highly sensitive design of a nanocavity resonator design based on metal -insulator-metal waveguide scheme. The cavity is in the form of L-shape which provides strong electric field confinement at resonance wavelength. The geometric parameters such as cavity length strongly manipulate the resonance wavelength of the sensor whereas the variation in the gap between the bus waveguide and the cavity seems to have less control over the resonance wavelength. The numerical analysis of the sensor designs is performed via the finite element method (FEM). To further enhance the performance of the sensor, we placed an array of nanoblocks in the regions of high concentration of electric field distribution in the cavity. This results in the intensification of the energy in the cavity which elevates the level of light-matter interaction. The proposed sensor design is simple yet provides very high sensitivity, FOM and $Q$-factor at the same time. The numerically calculated values of sensitivity, FOM and $Q$-factor of $791.67 \mathrm{~nm} / \mathrm{RIU}, 184.53$ and 255.71 are obtained for the standard resonator design, respectively. However, nanoblocks embedded design offers sensitivity, FOM and $Q$-factor of $1065 \mathrm{~nm} / \mathrm{RIU}, 251.17$ and 343.39, respectively, which is higher than most of the previously reported works.

Acknowledgements - This work was financially supported by the Ministry of Science and Higher Education of the Russian Federation (the scientific state assignment No. 0777-2020-0017 of the Samara National Research University) for numerical calculations, by the FSRC "Crystallography and Photonics" of the Russian Academy of Sciences (the state task No. 007-GZ/Ch3363/26) for theoretical research, and by the Russian Science Foundation (grant No. 20-69-47110) for comparative analysis. Also, this work was done within the framework of "Hybrid sensor platforms of integrated photonic systems based on ceramic and polymer materials" project is carried out within the TEAM-NET programme of the Foundation for Polish Science financed by the European Union under the European Regional Development Fund, POIR.04.04.00-00-14D6/18-01.

\section{References}

[1] Berini P., De Leon I., Surface plasmon-polariton amplifiers and lasers, Nature Photonics 6, 2012, pp. 16-24, DOI: 10.1038/nphoton.2011.285.

[2] Barnes W.L., Dereux A., EbBesen T.W., Surface plasmon subwavelength optics, Nature 424, 2003, pp. 824-830, DOI: 10.1038 /nature01937.

[3] Zhu W., Xu T., Wang H., Zhang C., Deotare P.B., Agrawal A., Lezec H.J., Surface plasmon polariton laser based on a metallic trench Fabry-Perot resonator, Science Advances 3(10), 2017, article e1700909, DOI: $10.1126 /$ sciadv.1700909.

[4] Cselyuszka N., Sakotic Z., Kitic G., Crnojevic-Bengin V., Jankovic N., Novel dual-band band-pass filters based on surface plasmon polariton-like propagation induced by structural dispersion of substrate integrated waveguide, Scientific Reports 8, 2018, article 8332, DOI: 10.1038/s41598-018 -26705-w.

[5] Wu L., GuO J., XU H., DAI X., XIANG Y., Ultrasensitive biosensors based on long-range surface plasmon polariton and dielectric waveguide modes, Photonics Research 4(6), 2016, pp. 262-266, DOI: 10.1364/PRJ.4.000262. 
[6] Zheng L., Zywietz U., Evlyukhin A., Roth B., Overmeyer L., Reinhardt C., Experimental demonstration of surface plasmon polaritons reflection and transmission effects, Sensors 19(21), 2019, article 4633, DOI: $10.3390 / \mathrm{s} 19214633$.

[7] Butt M.A., Khonina S.N., KaZAnskiy N.L., Ultra-short lossless plasmonic power splitter design based on metal-insulator-metal waveguide, Laser Physics 30(1), 2020, article 016201, DOI: 10.1088/ $1555-6611 / \mathrm{ab} 5577$.

[8] Passinger S., Seidel A., Ohrt C., Reinhardt C., Stepanov A., Kiyan R., Chichkov B.N., Novel efficient design of Y-splitter for surface plasmon polariton applications, Optics Express 16(19), 2008, pp. 14369-14379, DOI: 10.1364/OE.16.014369.

[9] Tong L., Wei H., Zhang S., Xu H., Recent advances in plasmonic sensors, Sensors 14(5), 2014, pp. 7959-7973, DOI: 10.3390/s140507959.

[10] Choo H., Kim M.K., Staffaroni M., Seok T.J., Bokor J., Cabrini S., Schuck P.J., Wu M.C., YablonOvitch E., Nanofocusing in a metal-insulator-metal gap plasmon waveguide with a three -dimensional linear taper, Nature Photonics 6, 2012, pp. 838-844, DOI: 10.1038/nphoton.2012.277.

[11] Anous N.H., KhaliL D.A., Performance evaluation of a metal-insulator-metal surface plasmon resonance optical gas sensor under the effect of Gaussian beams, Applied Optics 53(11), 2014, pp. 2515 -2522, DOI: 10.1364/AO.53.002515.

[12] Kong Y., Wei Q., Liu C., Wang S., Nanoscale temperature sensor based on Fano resonance in metal-insulator-metal waveguide, Optics Communications 384, 2017, pp. 85-88, DOI: 10.1016/ j.optcom.2016.09.041.

[13] Wang L., Zeng Y.-P., Wang Z.-Y., XIA X.-P., Liang Q.-Q., A refractive index sensor based on an analogy T shaped metal-insulator-metal waveguide, Optik 172, 2018, pp. 1199-1204, DOI: 10.1016 / j.ijleo.2018.07.093.

[14] Rakhshani M.R., Optical refractive index sensor with two plasmonic double-square resonators for simultaneous sensing of human blood groups, Photonics and Nanostructures - Fundamentals and Applications 39, 2020, article 100768, DOI: 10.1016/j.photonics.2020.100768.

[15] Li X., Zhang Z., Guo F., Huang Y., Zhang B., Zhang L., Yang Q., Tan Y., Liu X., Bai H., Song Y., Tunable plasmonically induced reflection in HRR-coupled MIM waveguide structure, Optik 199, 2019, article 163353, DOI: $10.1016 /$ j.ijleo.2019.163353.

[16] Yan S., Zhang M., Zhao X., Zhang Y., Wang J., Jin W., Refractive index sensor based on a metal -insulator-metal waveguide coupled with a symmetric structure, Sensors 17(12), 2017, article 2879, DOI: $10.3390 / \mathrm{s} 17122879$.

[17] KaZanskiy N.L., Khonina S.N., Butt M.A., Plasmonic sensors based on metal-insulator-metal waveguides for refractive index sensing applications: a brief review, Physica E 117, 2020, article 113798, DOI: 10.1016/j.physe.2019.113798.

[18] Butt M.A., Khonina S.N., KaZAnskiy N.L., A multichannel metallic dual nano-wall square split-ring resonator: design analysis and applications, Laser Physics Letters 16(12), 2019, article 126201, DOI: $10.1088 / 1612-202 X / a b 5574$.

[19] Butt M.A., KAZANSKiY N.L., KHONina S.N., Label-free detection of ambient refractive index based on plasmonic Bragg gratings embedded resonator cavity sensor, Journal of Modern Optics 66(19), 2019, pp. 1920-1925, DOI: 10.1080/09500340.2019.1683633.

[20] Butt M.A., Khonina S.N., KaZAnskiY N.L., An array of nano-dots loaded MIM square ring resonator with enhanced sensitivity at NIR wavelength range, Optik 202, 2020, article 163655, DOI: 10.1016/j.ijleo.2019.163655.

[21] Butt M.A., Khonina S.N., KaZAnskiy N.L., Metal-insulator-metal nano square ring resonator for gas sensing applications, Waves in Random and Complex Media 31(1), 2021, pp. 146-156, DOI: $10.1080 / 17455030.2019 .1568609$.

[22] Gai H., Wang J., Tian Q., Modified Debye model parameters of metals applicable for broadband calculations, Applied Optics 46(12), 2007, pp. 2229-2233, DOI: 10.1364/AO.46.002229. 
[23] Naghizadeh S., Kocabas S.E., Guidelines for designing 2D and 3D plasmonic stub resonators, Journal of the Optical Society of America B 34(1), 2017, pp. 207-217, DOI: 10.1364/JOSAB.34.000207.

[24] YIN J., TIAN J., YANG R., Investigation of the transmission properties of a plasmonic MIM waveguide coupled with two ring resonators, Materials Research Express 6(3), 2019, article 035018, DOI: 10.1088/2053-1591/aaf483.

[25] Zhang Z., Luo L., Xue C., Zhang W., Yan S., Fano resonance based on metal-insulator-metal waveguide coupled double rectangular cavities for plasmonic nanosensors, Sensors 16(5), 2016, article 642, DOI: $10.3390 / \mathrm{s} 16050642$.

[26] ZAFAR R., SALIM M., Enhanced figure of merit in Fano resonance-based plasmonic refractive index sensor, IEEE Sensors Journal 15(11), 2015, pp. 6313-6317, DOI: 10.1109/JSEN.2015.2455534.

[27] Jankovic N., Cselyuszka N., Multiple Fano-like MIM plasmonic structure based on triangular resonator for refractive index sensing, Sensors 18(1), 2018, article 287, DOI: 10.3390/s18010287.

[28] Shi Y., Zhang G.-M., An H.-L., Hu N., Gu M.-Q., Controllable Fano resonance based on coupled square split-ring resonance cavity, Guangzi Xuebao/Acta Photonica Sinica 46(4), 2017, article 0413002.

[29] Santis C.T., Stegger S.T., Vilenchik Y., Vasilyev A., Yariv A., High-coherence semiconductor lasers based on integral high-Q resonators in hybrid Si/III-V platforms, Proceedings of the National Academy of Sciences of the United States of America, PNAS 111(8), 2014, pp. 2879-2884, DOI: $10.1073 /$ pnas. 1400184111.

[30] Karimzadeh-Jazi R., Honarvar M.A., Khajeh-Khalili F., High Q-factor narrow-band bandpass filter using cylindrical dielectric resonators for X-Band applications, Progress In Electromagnetics Research (PIER) Letters 77, 2018, pp. 65-71, DOI: 10.2528/PIERL18041007.

[31] Borja A.L., Belenguer A., Esteban H., Boria V.E., Design and performance of a high-Q narrow bandwidth bandpass filter in empty substrate integrated coaxial line at $K_{u}$-band, IEEE Microwave and Wireless Components Letters 27(11), 2017, pp. 977-979, DOI: 10.1109/LMWC.2017.2750118.

[32] Uesugi T., Song B.-S., Asano T., Noda S., Investigation of optical nonlinearities in an ultra-high- $Q$ Si nanocavity in a two-dimensional photonic crystal slab, Optics Express 14(1), 2006, pp. 377-386, DOI: 10.1364/OPEX.14.000377.

[33] Chrostowski L., Grist S., Flueckiger J., Shi W., Wang X., Ouellet E., Yun H., Webb M., Nie B., Liang Z., Cheung K.C., Schmidt S.A., Ratner D.M., JAeger N.A.F., Silicon photonic resonator sensors and devices, Proceedings of SPIE 8236, 2012, article 823620, DOI: $10.1117 / 12.916860$.

Received February 26, 2020

in revised form March 28, 2020 